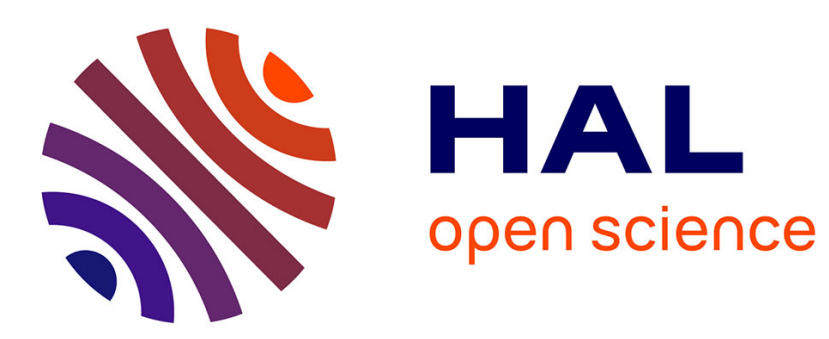

\title{
Les conditionnels potentiel et irréel en breton: De la morphologie temporelle à la valeur instructionnelle
}

Didier Bottineau

\section{To cite this version:}

Didier Bottineau. Les conditionnels potentiel et irréel en breton: De la morphologie temporelle à la valeur instructionnelle. Faits de langues, 2012, 40, pp.85-92. halshs-00922746

\section{HAL Id: halshs-00922746 \\ https://shs.hal.science/halshs-00922746}

Submitted on 30 Dec 2013

HAL is a multi-disciplinary open access archive for the deposit and dissemination of scientific research documents, whether they are published or not. The documents may come from teaching and research institutions in France or abroad, or from public or private research centers.
L'archive ouverte pluridisciplinaire HAL, est destinée au dépôt et à la diffusion de documents scientifiques de niveau recherche, publiés ou non, émanant des établissements d'enseignement et de recherche français ou étrangers, des laboratoires publics ou privés. 


\title{
Les conditionnels potentiel et irréel en breton : De la morphologie temporelle à la valeur instructionnelle
}

\author{
Didier Bottineau*
}

\section{VALEUR INSTRUCTIONNELLE DES FORMES DES CONDITIONNELS BRETONS}

Dans diverses langues romanes et germaniques, les marques conditionnelles des verbes se présentent sous des formes composites comparables : un marqueur d'ultériorité, $-r$ infinitival dans les langues romanes ou un auxiliaire lexical dans les langues germaniques (allemand werden «devenir », anglais will 'futur' par dématérialisation de willan «vouloir »), se combine à un marqueur issu du latin habere au passé (langues romanes: imparfait, français -ait, espagnol -ía, sauf italien : passé simple -ebbe ; dans les langues germaniques un marqueur analogue de passé est obtenu par apophonie et/ou finale dentale du prétérit (anglais would), avec en allemand modalisation par le Umlaut (werden «devenir», prétérit wurde, conditionnel würde). Dans le cadre d'une perspective instructionnelle (Bottineau 2010b, Col \& al. 2010), on part du principe que l'invariant interprétatif de la forme dépend (i) de sa composition submorphémique et (ii) du réseau d'oppositions dans lequel s'inscrit l'opérateur et, éventuellement, les submorphèmes qui le composent. S'appuyant sur ces indicateurs, divers travaux ont vu dans le conditionnel un ultérieur du passé référentiel, énonciatif ou dialogique, en établissant un rapport direct entre les valeurs contextuelles observée et la contribution de la submorphologie. Dans cette optique, le breton offre un cas remarquable avec la cohabitation de deux formes simples du conditionnel, communément nommées (conditionnels) potentiel et irréel. En indépendante, leurs emplois recoupent ceux du conditionnel français (atténuation, emprunt). En phrase conditionnelle, ils apparaissent tous deux en protase et/ou apodose à la manière du subjonctif II allemand, avec des appariements variés non réductibles à des corrélations mécaniques. En complétive à caractère énonciatif (dire, croire) et/ou modal (vouloir, craindre) ils alternent et se prêtent à diverses « concordances variables», chevauchant les emplois du conditionnel et du subjonctif romans. En système, ils cohabitent avec un « futur/subjonctif » unique (utilisable en indépendante et en subordonnée, compris comme futur ou comme subjonctif en subordonnée).

* CNRS, MoDyCo, Université Paris Ouest, Courriel : didier.bottineau@u-paris10.fr 
Morphologiquement, tous deux présentent une composition, distincte de celle des conditionnels romans : potentiel $-f e=$ subjonctif archaïque $f+$ imparfait $-e^{l}$; irréel -je (issu d'un ancien plus-que-parfait) = passé simple $-j{ }^{2}+$ imparfait $-e$. Tous deux intègrent l'élément imparfait ${ }^{3}$, fonctionnellement analogue à l'imparfait unique -ait / -ía des langues romanes. Mais seul le potentiel possède un marqueur assimilable à un opérateur d'ultériorisation, le subjonctif archaïque $f$ - de - fe. Pour l'irréel, la rencontre de deux marqueurs de passé, l'un imperfectif $e$ et l'autre perfectif $-j$, a de quoi déconcerter. La question est donc de déterminer si la sémantique et pragmatique du potentiel doit un élément d'ultériorisation à la présence de l'élément subjonctif, et de préciser la valeur instructionnelle de l'irréel avec sa composition originale. D'un point de vue contrastif, la composition morphémique des conditionnels bretons est analogue à celles des conditionnels uniques du français et de l'espagnol : le couple $f / j$ responsable de la valeur hypothétique joue un rôle analogue à celui du morphème - $r$-roman, qui au demeurant entre aussi dans la composition de l'un des subjonctifs imparfaits castillans (cantar «chanter», cantará « chantera», cantara «chantât»), et la marque d'imparfait correspond terme à terme. Les valeurs d'emploi des conditionnels du breton montrent que l'effet du marqueur d'imparfait est dans son principe comparable à celui de son homologue français: le renvoi de l'hypothèse à une source énonciative antériorisée. Ce qui intrigue, c'est l'alternance, en lieu et place du marqueur $-r$ - d'ultériorisation, des marqueurs $-f$ et $-j-$.

Le premier, $-f$ - subjonctif, figure un possible envisagé prospectivement, en attente d'actualisation, et marque une orientation positive vers un actualisable. Si l'on replace ce marqueur dans le dispositif de la chronogenèse de Guillaume (1929), là où les langues romanes inscrivent dans le conditionnel une marque $-r$ issue du mode quasi-nominal (l'infinitif), le breton inscrit un marqueur subjonctif $-f-$, toujours en amont du niveau de l'indicatif.

Le second, -j- du révolu temporel (passé simple) et aspectuel (plus-queparfait), figure un possible envisagé rétrospectivement, au-delà du seuil de réalisabilité, révolu, en principe trop tardif pour être concrétisé. Il se traduit parfois par un conditionnel parfait (aurait + participe passé) mais pas nécessairement (il peut s'agir d'un évènement dont l'idée de la réalisabilité n'est plus acceptable même si l'évènement lui-même n'est pas repéré comme passé).

Cette distinction devrait en principe suffire à caractériser les invariants des conditionnels : $-f e$ - repère le dire d'un énonciateur antérieur $(-e)$ comme l'avant

\footnotetext{
${ }^{1}$ Humphreys (1985, 369-373) signale que dans le parler de Bothoa ce marqueur peut être précédé d'un schwa, obligatoire pour certains verbes, inattesté pour d'autres, en variation «apparemment libre » pour d'autres encore ; Favereau (1997) y voit un simple marqueur d'appui.

${ }^{2}$ Le passé simple est peu ou pas usité en breton parlé, mais bien représenté dans les textes littéraires écrits; le marqueur - $j$ - encore observable à la première personne du pluriel skrivjomp «écrivîmes ». Le détail de l'évolution de ces formes en diachronie, ainsi que des éléments de variation dialectale, sont donnés par Hewitt 2010 et 2011.

3 La présence du - $e$ d'imparfait dans - $f e$ est mentionnée par certaines grammaires pédagogiques du breton, comme Desbordes $(1999,64)$.
} 
de l'actualisation visée $(f-)$ et indique une hypothèse antérieure et prospective, en amont de la réalisation, de concrétisation négociable; et -je- repère ce dire comme l'après et indique une hypothèse postérieure, une suggestion rétrospective figurée comme révolue ou rétroactive, donc inactualisable.

Cette analyse marcherait parfaitement si le conditionnel dit irréel n'exprimait toujours que, justement, l'irréel. En fait, il s'utilise aussi, paradoxalement, pour des hypothèses dont le contenu est présenté comme allant de soi (en tant que suggestion) pour les deux interlocuteurs et non pas comme une vue de l'esprit dont le locuteur serait la source subjective exclusive. Ceci rend nécessaire l'ajout d'un critère d'analyse, l'allocutivité : les marqueurs submorphémiques d'antériorité et postériorité sont également utilisés comme indicateurs d'incomplétude et de complétude interlocutive, -fe-produisant une hypothèse négociable dans le dialogue, et -je- une hypothèse conclusive et fermée, soit parce que sa réalisation est bloquée, soit parce que son contenu est conventionnel, voire les deux à la fois. La construction complexe instruite par les submorphèmes régit conjointement (i) la chronologie évènementielle de l'hypothèse, (ii) la chronologie énonciative du rapport locuteur / énonciateur, et (iii) la chronologie de la coordination interlocutive autour de la validation de l'hypothèse : elle porte sur la relation qui unit ces facettes et varie en contexte.

Ceci est corroboré par les gloses données par nombre de grammaires du breton, qui régulièrement invoquent l'accord interlocutif pour justifier les emplois de l'irréel. On propose donc que cette alternance articule deux discussions, (i) l'une d'ordre modal, le repérage d'un possible en amont ou en aval de l'actuel, (ii) l'autre d'ordre interlocutif, la négociation des points de vue en termes de convergence à établir ou obtenue; et que ces deux discussions impliquent l'aspectualisation de l'interlocution par la chronologie modale.

\section{REALISATIONS CONTEXTUELLES}

\subsection{Conditionnel atténuatif}

En breton, la forme correspondant au français « je voudrais » est normalement le conditionnel potentiel en $-f e$, utilisée lorsque la demande du locuteur se présente comme réalisable et s'ouvre prospectivement à l'approbation de l'allocutaire :

(1) Un hanter vagetenn a garfen kaout, posupl eo ?

'une demi-baguette je voudrais avoir, possible il est?'

« Je voudrais une demi-baguette, c'est possible?»

Plus rarement utilisé, l'irréel en -je- renvoie à l'accompli -j- la possibilité d'actualiser le procès attribué à l'énonciateur distancié par - $e$ - : il produit une hypothèse présentée comme périmée, révoquée, et pour laquelle l'avis de l'allocutaire n'est pas sollicité. On s'attendrait donc à ne jamais trouver la formulation a garjen pour les demandes. On en trouve toutefois des occurrences 
sporadiques, chez les classiques comme en breton parlé actuel, lorsque le désir exprimé s'affiche comme irréaliste et n'appelle pas la corroboration interlocutive :

(2) Piv zo barrek da cheñch skridoù en ur skeudenn ? Sed amañ ar skeudenn a garjen treiñ ar gerioù enni : Chromosome Turkish Text.png hag ar gerioù : cekirdek: nukleus hücre: kellig telomer: telomer sentromer: santromer kromatit : kromatit histon : histon çift sarmal : biñs doubl TDN baz çiftleri «Qui est capable de traduire le texte d'une image ? Voici l'image dont j'aimerais traduire le texte: Chromosome Turkish Text.png et le texte: çekirdek: nukleus hücre: kellig telomer: telomer sentromer: santromer kromatit : kromatit histon : histon çift sarmal : biñs doubl TDN baz çiftleri »

Avec une subordonnée, on s'attend à un accord ou une harmonie des modalités :

(3) Me a garje e vije meur a verour oc'h ober war-dro, da glask kaout un emglev ledan.

«J'aimerais qu'il y ait plus de fermiers qui s'y mettent dans le secteur, pour essayer d'avoir une large entente. »

Cet usage était déjà en vigueur au $19^{\mathrm{e}}$ siècle, comme dans la complainte de Marguerite Le Soaz: quand elle déclare à l'irréel Me a garje bezañ marv «j'aimerais être morte»- une évidence dans sa situation (contexte : elle préfèrerait mourir plutôt que de renoncer à soigner son père lépreux), son père lui répond au potentiel Perak karfac'h be'añ marvet? "Pourquoi voudrais-tu être morte ? », contestant l'évidence de l'idée. L'irréel signifie ici la non-réalité du procès ; le potentiel du père ne remet pas en cause ce statut, mais réattribue cette vision au point de vue subjectif de sa fille, qu'il ne partage pas: le potentiel rompt le consensus interlocutif initialement posé par elle.

Lorsque le locuteur avance une suggestion prudente « Ce serait bien de / si », il emploie le plus souvent le potentiel, pour une suggestion à laquelle le locuteur croit relativement, qu'il présente à l'allocutaire pour mise en discussion :

(4) Mat e vefe brezhonekaat ar forom-mañ evel just

'bien que serait bretonniser le forum-ci comme juste'

«Evidemment ce serait bien parler breton sur ce forum. »

Après cette forme, on attend dans le dialogue la poursuite de la discussion sur le thème de la suggestion lancée comme une bouteille à la mer : les membres du forum sont invités à soutenir la proposition, mais le potentiel prévoit formellement que l'opposition est possible, ménageant pour tout lecteur un espace de liberté pour déterminer sa propre position. Le potentiel fonctionne comme un imperfectif énonciatif dans l'interlocution en ce qu'il produit une contribution incomplète en attente de perfectivation ultériorisée. Le complément de construction du sens doit venir du partenaire dialogal matériel ou possible (les lecteurs auxquels s'adresse un texte) et son contenu tourner autour de l'acceptation ou du refus de l'actualisation. Dans ce type de contexte, le conditionnel irréel produit l'effet inverse : 
(5) Met mat e vije sikour muioc'h ar re o deus prenet tourioù-avel.

'mais bien que serait secourir plus les gens leur est acheté tours-vent'

«Mais ce serait bien d'aider davantage ceux qui ont acheté des éoliennes. »

Selon les contextes, cet énoncé peut signifier que la suggestion est irréaliste et/ou que tout lecteur est censé adhérer à son contenu (ce qui n'est nullement contradictoire!)

Pour le conditionnel d'emprunt, cette distribution pose problème ( $D e$ nouveaux témoignages accableraient Servier sur le Mediator) : dans la presse bretonnante actuelle on observe bien cet emploi, sans doute par fait de contact, le plus souvent avec la forme en $-f e$, parfois celle en -je (comme le signale Hewitt $2010,303)$. Le problème est que dans cet emploi l'hypothèse n'émane pas du locuteur (qui invoque un ouï-dire) et n'est pas partagée avec l'allocutaire, dont l'avis n'est pas sollicité en renfort: aucune des deux formes ne convient réellement dans son fonctionnement systémique, et on constate une hésitation dans ces emplois d'origine étrangère.

\subsection{Protases et apodoses}

Les deux conditionnels sont utilisés dans les deux parties de la phrase conditionnelle. Le précis de syntaxe de Le Gléau (1973) stipule que « La logique exige que protase et apodose adoptent le même type de formes conditionnelles. Ainsi en usent les classiques : Scol ar Marv Mad (1864) contient 31 phrases conditionnelles : 7 entièrement à l'irréel, 24 entièrement au potentiel. Aucune n'admet l'alternance incorrecte : Deufe / Bije ou En dije / karfe, usuelle en parlers KLT. » La grammaire prescriptive requiert que les conditionnels utilisés dans le couple protase / apodose soient harmonisés pour des raisons d'ordre logique, mais l'usage attesté n'a jamais appliqué ce principe. Dans la protase comme dans l'apodose, on trouve l'un ou l'autre des conditionnels, le choix spécifiant le caractère interlocutivement ouvert ou fermé de l'éventualité correspondant à la condition ou à la conséquence :

Protase au potentiel + apodose au potentiel :

La condition est un possible ouvert par le locuteur, la conséquence également.

(6) Pegen brave vefe ma c'hallfe kalz reou all mont d'e heul

'combien beau que serait si pouvai_beaucoup autres aller à sa suite'

«Qu'est-ce que ce serait bien si beaucoup d'autres pouvaient l'imiter! »

Protase à l'irréel, apodose au potentiel :

La condition est présentée comme irréaliste ou allant de soi, alors que la conséquence relève de l'avis du locuteur sur la base posée dans la protase ${ }^{4}$. Il n'y

${ }^{4}$ Exemple de Kervella [1947] 1995, 183 : m'am bije arc'hant e prenfen an dra-se diouzhtu (hogen, n'em eus ket arc'hant) "si j'avais de l'argent j'achèterais cette choselà tout de suite (seulement, je n'ai pas d'argent) » (ma traduction) vs m'am befe arc'hant 
a pas de corrélation logique entre la protase et l'apodose : on peut suggérer une action possible et militer en faveur de son contenu en assumant subjectivement sa validité (apodose au potentiel) tout en l'adossant à une condition irréalisable (protase à l'irréel) ; cela revient à garantir que l'on réaliserait vraiment le projet si la condition n'était pas bloquée :

(7) Ma ouijen skoazellañ e rafen n'eus forzh petra

'Si je pouvais épauler je ferais n'est force quoi'

«Si je pouvais aider je ferais l'impossible.»

Protase au potentiel, apodose à l'irréel :

La protase reçoit une condition envisagée par le locuteur, et l'apodose une conséquence allant de soi, présumée comme indiscutable. Cette combinaison apparaît surtout quand l'apodose précède la protase :

(8) Ma zad neuze ma mamm na vijent ket kontan ma yafen me ganeoc'h.

"Mon père et ma mère ne seraient pas contents si je partais avec toi. »

(Ex. de Jules Gros 1989, Le trésor du breton parlé, expressions recueillies à

Trédrez-Loquémeau, près de Lannion, entre 1912 et 1968)

Protase et apodose à l'irréel :

La condition est irréalisable et l'effet aussi, et/ou l'effet va de soi.

(9) Mac'halljen mont ez ajen.

«Si je pouvais partir, je partirais. »

(titre de la leçon 38 de la méthode Ni a gomz Brezhoneg «nous parlons breton »).

Avec ha pa 'et quand' = «quand bien même », toujours suivi de l'un des conditionnels (même combiné à un futur en apodose) :

- Hypothèse recevable (potentiel), conséquence garantie :

(10) Ha pa spontfe ar re all, me ne spontin ket.

'Et quand s'effraieraient les autres, moi ne m'effraierai pas.'

« Même si les autres ont peur, moi je n'aurai pas peur. »

- Hypothèse irrecevable (irréel), conséquence fictive :

(11) Ha spi hoc'h eus da gavout ho preur?

«Et vous avez l'espoir de retrouver votre frère?»

Spi ebet. Ha pa'm bije bet spi, e vije aet da get pell 'zo.

«Aucun espoir. Et même si j'en avais eu, je l'aurais perdu depuis longtemps. »

e prenfen... (gellout a ran c'hoaz kaout arc'hant) «si j'avais assez d'argent achèterais... » (il est encore possible que j'aie de l'argent). Le Gléau $(2000,178)$ : «On emploie les formes irréelles pour exprimer l'hypothèse donnée comme contraire à une réalité dûment vérifiée ». 

instructionnelle

Dans l'exemple suivant, extrait d'un précis d'astronomie récent destiné au grand public, une comparaison est introduite par «imaginons que ». Dans la première phrase, le premier irréel vije «soit», régi par empennomp «imaginons », équivaut à un subjonctif français, et ce passage équivaut à une protase : «imaginons que les choses soient ainsi »= «si les choses étaient ainsi », avec en breton l'irréel dans les deux cas (empennomp «imaginons » est à l'impératif). Les phrases suivantes, introduites par Neuze «Alors », ainsi que le second potentiel de la première phrase he dije "elle aurait », développent une série d'apodoses, avec plusieurs verbes au conditionnel irréel pour énumérer une série de conséquences manifestement contrefactuelles. Le choix de ce mode permet au rédacteur d'expliciter le caractère fictif de la comparaison imaginée et interdit au lecteur d'imaginer un seul instant que l'auteur envisage sérieusement une telle possibilité et qu'il faille réfléchir à l'éventualité de sa réalisation.

(12) Evit kaout ur soñj eus ment Koskoriad en Heol e-keñver hini ar c'halaksienn, empennomp e vije hor galaksienn a-vent gant Frañs, da lavaret eo he dije $1000 \mathrm{~km}$ treuzkiz. Neuze e vije Koskoriad en Heol a-vent gant ur pezh ur santim euro. Gant ar binvioù modernañ zo ganimp evit beajiñ en egor, e vije ezhomm eus 15 vloaz d'un astraer da vont eus an Douar (kreiz hor pezh 1 santim euro) betek Ploudon (lezenn ar pezh). Evit tizhout ar steredenn dostañ d'an Heol (Alpha Centauri) war-hed 43 metr, ez istimer e vije ezhomm eus ur veaj 100000 bloaz., hag evit treuriñ ar c'halaksienn e vije ezhomm eus un hebeud miliardoù a vloavezhioù.

«Pour nous faire une idée de la taille du système solaire par rapport à celle de la galaxie, imaginons que notre galaxie soit de la taille de la France, c'est-àdire qu'elle aurait $1000 \mathrm{~km}$ de diamètre. Alors notre Système Solaire serait de la taille d'une pièce d'un euro. Avec les moyens les plus modernes dont nous disposons pour voyager dans l'espace, il faudrait plus de 15 ans pour qu'un astronaute se rende de la Terre (le centre de notre pièce) à Pluton (le bord de la pièce). Pour atteindre l'étoile la plus proche du Soleil (Alpha Centauri) distante de 43 mètres, on estime qu'il faudrait un voyage de 100000 ans, et pour traverser la galaxie il faudrait plusieurs milliards d'années. »

Cette distinction grammaticale entre une hypothèse à prendre au sérieux (potentiel) et une hypothèse à prendre comme un jeu de l'esprit (irréel), caractéristique de la tendance du breton à formaliser en morphosyntaxe les conditions d'interactions entre interlocuteurs, a existé en français avec le subjonctif imparfait (fussé-je riche, l'eussé-je su) et disparu hormis certains figements employés par ironie ou auto-dérision, généralement sans produire l'effet précis que cette construction devait susciter. Les exemples qui précèdent attestent de la productivité du système en breton dans des contextes d'expression orale et écrite relativement populaires. L'exemple astronomique montre en particulier que le choix de l'irréel pour les différents verbes a fait l'objet d'une motivation commune indépendante des insertions syntaxiques (complétive pour le premier, pseudo-apodoses pour les suivants). Dans les autres types de subordonnées, que nous laissons ici de côté, le fonctionnement est analogue avec des spécificités locales. 


\section{CONCLUSION}

Les deux conditionnels du breton permettent de distinguer deux modes de conceptualisation de l'hypothèse, l'une munie d'un marqueur d'ultériorisation pour une hypothèse transitoire et ouverte à la renégociation par le dialogue, l'autre muni d'un marqueur de perfectivation pour une hypothèse conclusive et fermée à ce rapport. Les observables morphologiques suggèrent que ce contraste est obtenu par la mise en scène d'une configuration intersubjective complexe qui mobilise à la fois la profondeur énonciative et dialogique d'un côté et l'aspectualisation du rapport interlocutif de l'autre : la pondération en temps réel à l'instant de parole du niveau de progression auquel est parvenu un acte de conceptualisation que le locuteur partage avec l'allocutaire et la manière dont est planifié le positionnement attendu de l'allocutaire en termes d'adhésion et de poursuite.

\section{BIBLIOGRAPHIE}

Bottineau D., 2010a, Les temps du verbe breton: Temps, aspect, modalité, interlocution, cognition - des faits empiriques aux orientations théoriques, in C. Douay (ed), Système et chronologie, Rennes, Presses Universitaires de Rennes, 129-157.

--- 2010b, Language and enaction, in J. Stewart, O. Gapenne, E. Di Paolo (eds), Enaction: toward a new paradigm for cognitive science, MIT, 267-306.

Bres J., 2009, «Dialogisme et temps verbaux de l'indicatif », Langue française $163,2009 / 3,21-39$.

Chalm E., 2008, La grammaire bretonne pour tous, An Alarc'h Embannadurioù / Editions Le Cygne, Lannion.

Claris J.-M. Notes sur les formes en -rait. Langue française 11, 1. Syntaxe transformationnelle du francais, 32-38.

Col G., Aptekman J., Girault S. \& Victorri B., 2010, Compositionnalité gestaltiste et construction du sens par instructions dynamiques, Cognitextes 5, Numéro spécial AFLiCo 3 Grammaires en Construction(s), consulté le 01/04/2011 : http://cognitextes.revues.org/372

Favereau F., 1997, Grammaire du breton contemporain, Skol Vreizh, Morlaix.

Guillaume G. [1929] 1965, Temps et verbe, Champion, Paris.

Kervella F., [1947] 1995, Yezhadur bras ar brezhoneg, Al Liamm.

Hémon R., [1940] 1975, Grammaire bretonne, $8^{\mathrm{e}}$ éd., Al Liamm, Brest.

Hewitt S., 2010, Mood in Breton, Rothstein B. \& Thieroff R. (eds), Mood in the Languages of Europe, John Benjamins, Amsterdam \& Philadelphia.

Hewitt S., 2011, A tale of two conditionals, Long version of "Mood in Breton", presented at the International Congress of Celtic Studies, Maynooth, 1-5 August 2011.

Humphreys H. L., 1985, Phonologie et morphosyntaxe du parler breton de Bothoa, Emglev Breizh, Brest.

Le Gléau R., 1973, Syntaxe du breton moderne (1710 - 1972), Editions La Baule.

Rocchetti A., 2003, Il condizionale in italiano e nelle lingue romanze, Società di Linguistica Italiana (SLI) 46 Il verbo italiano, 85-97. 